\title{
A RESULT ON POSITIVE MATRICES AND APPLICATIONS TO HANKEL OPERATORS
}

\author{
MARCUS CARLSSON
}

Abstract. Let $S$ denote the shift operator on $l^{2}(\mathbb{N})$ and set $e_{0}=(1,0,0, \ldots)$. A special case of the main result says that if $W$ is a self-adjoint operator on $l^{2}(\mathbb{N})$ such that $W\left(e_{0}\right)=0$ and $S^{*} W S \geqslant W$, then $W \geqslant 0$. We apply this result to AAK-type theorems on generalized Hankel operators, providing new insights related to previous work by S. Treil and A. Volberg [10].

Mathematics subject classification (2010): Primary 47B35, Secondary 47B15, 47B37.

Keywords and phrases: Positive matrices, Hankel operators.

\section{REFERENCES}

[1] V. M. Adamjan, D. Z. Arov, M. G. Krein, Analytic properties of the Schmidt pairs of a Hankel operator and the generalized Schur-Takagi problem (Russian), Mat. Sb. (N.S.), 86, 128 (1971), 34-75.

[2] A. Aleman, Finite codimensional invariant subspaces in Hilbert spaces of analytic functions, J. Funct. Anal., 119, 1 (1994), 1-18.

[3] J. R. ButZ, s-numbers of Hankel matrices, J. Functional Analysis, 15 (1974), 297-305.

[4] M. CARLSSOn, AAK-theory on weighted spaces, GMIG-project review report 2009, Purdue University.

[5] J.B. Conway, A course in functional analysis, Second edition, Graduate Texts in Mathematics, 96. Springer-Verlag, New York, 1990.

[6] C. FoiAs, S. Sz-NAGY, Harmonic analysis of operators on Hilbert space.Harmonic analysis of operators on Hilbert space, North-Holland Publishing Co., 1970.

[7] N. K. NiKOLSKI, Operators, functions, and systems: an easy reading. Vol. 1. Hardy, Hankel, and Toeplitz, Mathematical Surveys and Monographs, 92. American Mathematical Society, Providence, RI, 2002.

[8] V.V. Peller, Hankel operators and their applications, Springer Monographs in Mathematics, Springer-Verlag, New York, 2003.

[9] R. Rochberg, A new characterization of Dirichlet type spaces and applications, Illinois J. Math., 37, 1 (1993), 101-122.

[10] S. Treil, A. Volberg, A fixed point approach to Nehari's problem and its applications, 165-186, Oper. Theory Adv. Appl., 71, Birkhäuser, Basel, 1994. 